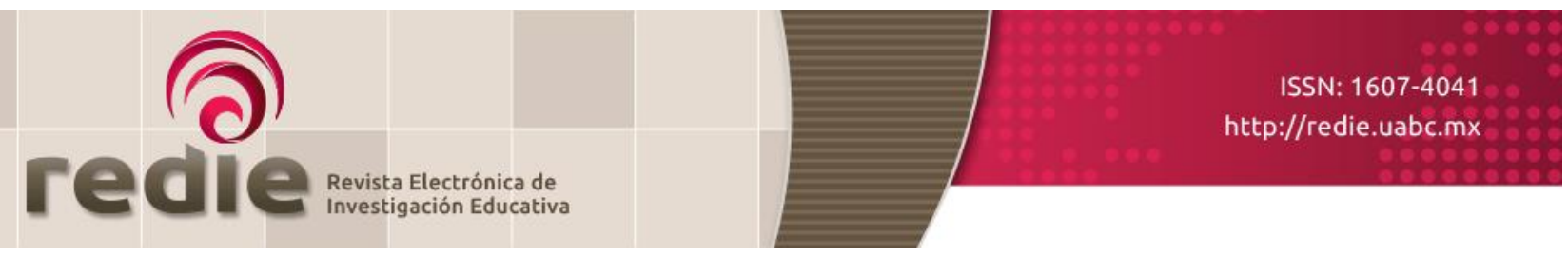

Vol. 19, Núm. 1, 2017

\title{
Nuevos modelos tecnopedagógicos. Competencia digital de los alumnos universitarios
}

\author{
New Techno-Pedagogical Models: Digital Competence \\ in University Students
}

\author{
Almudena Castellanos Sánchez (1) almudena.castellanos@unir.net \\ Cristina Sánchez Romero (2) csanchez@edu.uned.es \\ José Fernando Calderero Hernández (1) josefernando.calderero@unir.net \\ (1) Universidad Internacional de la Rioja \\ (2) Universidad Nacional de Educación a Distancia \\ (Recibido: 21 de abril de 2015; Aceptado para su publicación: 5 de agosto de 2015) \\ Cómo citar: Castellanos, A., Sánchez, C. y Calderero, J. F. (2017). Nuevos modelos tecnopedagógicos. Competencia digital de los \\ alumnos universitarios. Revista Electrónica de Investigación Educativa, 19(1), 1-9. Recuperado de \\ https://doi.org/10.24320/redie.2017.19.1.1148
}

\section{Resumen}

Este artículo muestra los resultados conseguidos mediante una investigación de carácter cuantitativo, realizada con el objetivo de conocer el perfil competencial en materia tecnológica de 301 estudiantes que ingresan por primera vez en el Grado universitario de Magisterio de Primaria. Se utilizó un cuestionario digital constituido por 29 preguntas; los resultados demuestran el predominio de alumnos mayores de 30 años, de sexo femenino, que disponen de ordenador con conexión a Internet, y cuyo medio favorito para interactuar es el teléfono, navegan a diario y emplean el correo electrónico, manejan de manera básica las tecnologías, sobre todo el paquete de ofimática, y no están familiarizados con el uso de herramientas actuales, como los blogs o marcadores sociales. Se concluye que estos alumnos no comparten los rasgos que se esperan de un nativo digital: producir, difundir y consumir cultura a través de Internet.

Palabras clave: Competencias digitales, innovación educativa, educación superior, sociedad de la información.

\section{Abstract}

This paper offers the results of a quantitative study carried out with the aim of defining the technological skills profile of 301 students entering the BA in elementary education. A 29-question digital survey was used; results reflect a prevalence of female students over 30 years of age, who have access to a computer with an Internet connection, prefer to interact using phones, use e-mail, surf the Internet every day, аге able to use technology at a basic level, particularly office suites, and are unfamiliar with the use of modern tools such as blogs and social bookmarking. It is concluded that these students do not display the characteristics expected of digital natives: the production, dissemination and consumption of culture online. 


\section{Introducción}

En los últimos tiempos hemos pasado de una sociedad que empleaba los símbolos gráficos para leer y escribir, a un nuevo modelo cultural que renueva el concepto de alfabetización, llevándolo más allá de la lectura y escritura textual, y que exige el dominio del lenguaje audiovisual y multimedia. La Universidad, en este sentido, se ve obligada a asumir una tarea alfabetizadora que haga posible el acceso igualitario a la tecnología y el desarrollo de una ciudadanía responsable y crítica (Área, Gutiérrez y Vidal, 2012). En la era de la información y el conocimiento, la alfabetización digital es un derecho del individuo.

Diferentes estudios apuntan a que en pocos años el perfil del alumno que ingresa en la universidad habrá cambiado, teniendo en cuenta que habrá vivido en una sociedad altamente tecnológica y entre sus características destacará una mayor inteligencia visual, preferencia por la hipertextualidad, por la inmediatez, por la flexibilidad, una mayor capacidad de resolución de problemas, capacidad de socialización en red, o por estar al día en cuanto a nuevos productos y servicios tecnológicos (Fundación Telefónica, 2012; Crue, 2012; Crue, 2012b). Por su parte, la Universidad se verá obligada a ajustarse a este nuevo perfil de alumno, trasformando desde herramientas y metodología hasta, en algunos casos, la forma de entender los procesos de enseñar y aprender.

Por otra parte, las tareas de la figura docente se verán igualmente afectadas por la nueva situación (Fundación Telefónica, 2012; Crue, 2012b). Los docentes tendrán que estar constantemente actualizando sus competencias (trabajo colaborativo internacional entre universidades en red, elaboración de contenidos en soporte tecnológico, gestión de la cantidad inabarcable de información en Internet...).

Una sociedad global exige de los docentes la actualización constante, deben aprender a manejar los entornos de formación en red, aprender a interactuar simultáneamente con un amplio volumen de estudiantes desde la virtualidad presencial, trabajar en colaboración con otros profesionales aprovechando las herramientas más nuevas para compartir y comunicarse, diseñar contenido interactivo y colaborativo, accesible desde cualquier dispositivo, etc.

En la actualidad coexisten diferentes paradigmas a la hora de hacer frente a la tarea de educar (Fundación Telefónica, 2012b). Sin embargo, cada vez más los centros educativos apuestan acertadamente por poner en el centro de sus acciones al alumno. Estos modelos que se están dando con fuerza en los centros educativos más innovadores, entienden la figura del docente como el responsable de diseñar los entornos que favorezcan el aprendizaje y aprovechan la tecnología como mediadora en la construcción del conocimiento y la interacción social, es lo que algunos autores han denominado pedagogías emergentes, haciendo referencia al conjunto de enfoques e ideas pedagógicas que están surgiendo alrededor del uso de las TIC en educación y que intentan aprovechar todo su potencial comunicativo, informacional, colaborativo, interactivo, creativo e innovador en el marco de una nueva cultura del aprendizaje (Adell y Castañeda, 2012).

Estos nuevos paradigmas educativos integran las tecnologías, tal es el caso del Technological Pedagogical Content Knowledge (TPCK), modelo que propone introducir las tecnologías en las aulas combinando los conocimientos pedagógicos del docente, sus conocimientos en cuanto al contenido que imparte y sus competencias tecnológicas (Mishra y Koehler, 2006; Cacheiro, 2011). Lo que se consigue bajo este modelo tecnopedagógico es integrar la tecnología con sentido. Sin embargo, no es éste un modelo exclusivo a la hora de integrar tecnología. Existe otra propuesta basada en los estilos de aprendizaje activo, teórico, reflexivo y pragmático- denominada Estilos de Aprendizaje y Actividades Polifásicas (EAAP), que permite incorporar las TIC en el aula teniendo en cuenta los estilos cognitivos que se quieren trabajar en el alumno, de tal forma que se personalice la educación ajustándose a la forma de aprender de cada uno y, a su vez, se fomenten nuevos estilos de aprendizaje (Lago, Colvin y Cacheiro, 2008; Cacheiro, 2011).

Otra pedagogía emergente que ha surgido con el uso de las tecnologías es la que se conoce como flipped classroom o aula invertida (Lage, Platt y Treglia, 2000; Bergmann y Sams, 2012). Los docentes que apuestan por este método aprovechan la presencialidad para interactuar con sus alumnos, trabajar en 
equipo, resolver dudas o colaborar en proyectos, y la virtualidad que permite la tecnología (videoconferencias, streaming, screencast, etc.) para impartir clases magistrales, de tal forma que el alumno pueda atender a los contenidos teóricos en cualquier momento y desde cualquier dispositivo, mientras que aprovecha la clase presencial para aprender de manera colaborativa con la resolución de dudas, trabajos de investigación, casos prácticos, debates, tutorías, etc. Sin embargo, no es ésta la única tendencia en pedagogía, actualmente se habla de cursos masivos y en línea (MOOc), aprendizaje ubicuo (ULearning), entornos personales de aprendizaje (PLE), y de modelos como Khan Academy o Bring Your Own Device (BYOD).

Pese a estas nuevas tendencias pedagógicas, algunos estudios (Pino y Soto, 2010; Prendes y Castañeda, 2010; Roig y Pascual, 2012) reflejan cómo el alumno universitario actual, aunque se va acercando, no pertenece aún a ese nuevo perfil de estudiante conectado, que domina la tecnología de su época y participa bajo una identidad digital construyendo cultura a través de la red (Reig y Vílchez, 2013). Es más bien un alumno que maneja el sistema operativo del ordenador, los paquetes de ofimática y las búsquedas por Internet, y de manera incipiente utiliza las redes sociales o publica en un blog.

\section{Método}

El objetivo de la investigación fue identificar las características de los alumnos que se matriculan en el Grado de Magisterio Primaria que oferta la Universidad Internacional de La Rioja, con el fin de conocer las competencias tecnológicas de las que parten, para desarrollar un plan de estudios que se ajuste a las tendencias pedagógicas emergentes propias de la era digital.

Acorde con el objetivo de analizar la situación de partida del alumno que ingresa en la universidad, recurrimos a métodos descriptivos con enfoque cuantitativo para especificar el perfil del estudiante que ingresa en la asignatura.

En el estudio participaron los alumnos matriculados en la asignatura "TIC aplicadas a la educación" del Grado Maestro de Educación Primaria de la Universidad Internacional de La Rioja. Un total de 301 estudiantes seleccionados a través de un muestreo no probabilístico intencional, por el que se escogieron a aquellos que cumplían el criterio de haber comenzado sus estudios en el periodo académico 2014-2015.

Dado que la investigación precisa encuestar a los sujetos del estudio para conocer y describir sus características, optamos por emplear un cuestionario digital. Esta técnica nos permitió amoldarnos a la condición de internacionalidad y distancia que asume la universidad, así como llegar a un mayor número de alumnos sin incrementar los costos del proyecto y tener la capacidad suficiente para recopilar la información sin lugar a sesgos (Ballester, 2001). A la hora de crearlo se han seguido los consejos de Fox (1989), se identifican los campos de contenido de la encuesta y las preguntas más relevantes en función de la finalidad del estudio, se analiza la muestra de individuos participantes en la investigación para poder establecer la mejor forma de acceder a ellos, se selecciona el tipo de pregunta a incluir, así como el modelo de respuesta, y se comprueba su validez.

El cuestionario quedó constituido por 29 preguntas que permitieron obtener información directa sobre el perfil de acceso de los estudiantes. Su organización (en cuatro secciones) hizo posible recabar datos de acuerdo con criterios de orden temático establecidos: datos personales (nombre, edad...), datos profesionales (experiencia en el campo de la educación, títulos universitarios anteriores...), competencias digitales (uso de internet, correo electrónico, paquete de ofimática...), motivación y expectativas (motivo por el que han elegido el grado, expectativas de la asignatura...). Esta disposición ayuda a evitar preguntas innecesarias y a cuidar la relación de éstas con el objetivo de la investigación. Las secciones se ordenaron de tal forma que despertaran el interés del informante, de lo más sencillo a lo más complejo (Hernández, 2004).

Para impedir que los alumnos matriculados en la asignatura rehusaran responderlo, las preguntas se plantearon de forma clara y precisa; por otro lado, para evitar que la persona que diera las instrucciones para cumplimentar el cuestionario influyera con su actitud en las respuestas, todos los estudiantes 
recibieron las mismas instrucciones por escrito, descargaron su cuestionario desde el propio campus virtual y lo enviaron a través del mismo medio. El hecho de que hayan podido cumplimentarlo a través de Internet tiene como ventaja, además, que el cuestionario se pueda aplicar a una muestra más amplia de alumnos, independientemente del país de procedencia. No obstante, con esta metodología se corría el riesgo de que algunos estudiantes no enviaran su respuesta. En este caso, se decidió que si el porcentaje era menor al $75 \%$ de respuestas, el profesor responsable insistiera en la importancia de su entrega para el buen funcionamiento de la asignatura.

La necesidad de validar este cuestionario se ha cumplido, por un lado, a través de la revisión del contenido por criterio de 15 expertos en la materia. El grupo de expertos evaluó con base a una escala Likert de 5 puntos la adecuación del cuestionario a la investigación, la comprensión de los ítems y la coherencia interna. Los resultados a este respecto señalaron la adecuación del cuestionario en los tres sentidos. No obstante, siguiendo el juicio de los expertos, se anuló alguna pregunta porque no afectaba al objetivo de la investigación, se reformuló alguna pregunta de tal forma que resultara más clara y se modificaron las opciones de respuesta que podían dar lugar a ambigüedades.

El producto es un cuestionario válido porque recoge los datos que necesitamos para la investigación, fidedigno porque cualquier investigador podría aplicarlo obteniendo los mismos resultados, y operativo, verificado por la experiencia (Pardinas, 2005). Por último, la prueba Alfa de Cronbach para medir la fiabilidad del cuestionario nos dice que el instrumento presenta una variabilidad homogénea, por encima del 0.8 (a301=0.87), considerada buena por George y Mallery (1995).

\section{Resultados}

El análisis estadístico de los resultados nos da la información que presentamos a continuación, agrupada en las cuatro secciones que conforman el cuestionario: datos personales, datos profesionales, competencias digitales y motivación y expectativas.

\subsection{Datos personales}

El intervalo de edad con mayor número de alumnos es el que va de los 30 a los 35 años ( $n=101 ; \%=33.6)$. Respecto a la variable sexo, el porcentaje más alto corresponde a la categoría mujer (75.4\%) como puede observarse en la tabla I. Si analizamos esta variable en relación a la edad, el intervalo de edad que recoge mayor número de mujeres es el de más de 57 años con un $85.7 \%$.

Tabla I. Frecuencias y porcentajes de la variable sexo

\begin{tabular}{lcccc}
\hline & Frecuencia & Porcentaje & $\begin{array}{c}\text { Porcentaje } \\
\text { válido }\end{array}$ & $\begin{array}{c}\text { Porcentaje } \\
\text { acumulado }\end{array}$ \\
\hline Hombre & 74 & 24.6 & 24.6 & 24.6 \\
Mujer & 227 & 75.4 & 75.4 & 100.0 \\
\hline Total & 301 & 100.0 & 100.0 & \\
\hline
\end{tabular}

\subsection{Datos Profesionales}

En cuanto a la situación laboral de los estudiantes que se matriculan en la asignatura, la gran mayoría trabaja actualmente $(n=244 ; \%=81.1)$, como se ve en la tabla II. 
Tabla II. Frecuencias y porcentajes de la variable trabaja actualmente

\begin{tabular}{lcccc}
\hline & Frecuencia & Porcentaje & $\begin{array}{c}\text { Porcentaje } \\
\text { válido }\end{array}$ & $\begin{array}{c}\text { Porcentaje } \\
\text { acumulado }\end{array}$ \\
\hline No & 57 & 18.9 & 18.9 & 18.9 \\
Sí & 244 & 81.1 & 81.1 & 100.0 \\
\hline Total & 301 & 100.0 & 100.0 & \\
\hline
\end{tabular}

\subsection{Competencias digitales}

A la pregunta de si el alumno tiene en casa ordenador con conexión a Internet, la mayoría (99.7\%) respondió que sí, siendo Windows el sistema operativo más empleado ( $n=276 ; \%=91.7)$. El medio que prefieren los estudiantes para contactar con los profesores es el Teléfono ( $n=280 ; \%=93$ ), seguido del Chat $(n=30 ; \%=10)$. En cuanto a la frecuencia con que los alumnos usan Internet, el $93.4 \%$ de ellos lo emplea a diario, y lo mismo ocurre con el correo electrónico $(n=244 ; \%=81.1)$. En cuanto al grado de conocimientos informáticos que consideran tener al comenzar la universidad, el $43.5 \%$ de los estudiantes dice estar en un nivel intermedio, un $42.2 \%$ sostiene que sus conocimientos son básicos, el $8 \%$ dice tener un conocimiento avanzado y $6.3 \%$ afirma que es principiante (ver tabla III).

Tabla III. Frecuencias y porcentajes variable conocimientos informáticos

\begin{tabular}{lcccc}
\hline & Frecuencia & Porcentaje & $\begin{array}{c}\text { Porcentaje } \\
\text { válido }\end{array}$ & $\begin{array}{c}\text { Porcentaje } \\
\text { acumulado }\end{array}$ \\
\hline Avanzado & 24 & 8.0 & 8.0 & 8.0 \\
Básico & 127 & 42.2 & 42.2 & 50.2 \\
Intermedio & 131 & 43.5 & 43.5 & 93.7 \\
Principiante & 19 & 6.3 & 6.3 & 100.0 \\
\hline Total & 301 & 100.0 & 100.0 & \\
\hline
\end{tabular}

En lo que se refiere al paquete de ofimática, el $45.8 \%$ de los estudiantes maneja el procesador de textos a nivel intermedio y el $28.2 \%$ lo hace a nivel básico; en cuanto al presentador de diapositivas, el 32.2\% lo maneja a nivel básico y el $24.3 \%$ a nivel intermedio; la hoja de cálculo es dominada a nivel básico por el $32.6 \%$ y el $25.6 \%$ tiene un nivel principiante. El porcentaje de alumnos que domina de forma avanzada estos programas es superior en el caso de los procesadores de textos (18.3\%), siendo más bajo en los programas el presentador de diapositivas (5\%) y la hoja de cálculo (4\%).

Preguntamos a los alumnos de primer curso del Grado Maestro Primaria que se han matriculado en la asignatura "TIC aplicadas a la educación", si manejan herramientas básicas de la era digital, como son los compresores de archivos (Winzip, Winrar, Zip...) a lo que el 70.8\% respondió que sí; sin embargo, el 70.4\% no domina los discos duros virtuales y sólo $14.3 \%$ sabe crear y gestionar un blog. Algo parecido ocurre con otros servicios 2.0, el $73.1 \%$ de los encuestados no sólo no maneja sino que además no conoce lo que son los marcadores sociales, el 51.2\% no conoce Google Sites, el 53.2\% no sabe lo que es Google Drive y el $68.4 \%$ desconoce lo que es Slideshare. En cuanto a programas tecnológicos que pudieran resultar de interés para el ámbito educativo, el $74.1 \%$ no conoce las webquest, el $90.7 \%$ no saben lo que son los programas para crear mapas conceptuales, el $85.3 \%$ no conoce el software de las pizarras digitales interactivas, el $84.7 \%$ no conoce programas para la edición de audio y el $77.8 \%$ no sabe lo que son las herramientas de autor.

\subsection{Motivaciones y expectativas}

Entre las motivaciones que han llevado a los alumnos a matricularse en el Grado en Magisterio de Primaria predomina la vocación docente $(n=244 ; \%=81.1)$, seguida de las salidas laborales ( $n=116$; $\%=38.5$ ). A través de una escala Likert con 5 valores se preguntó a los alumnos en qué medida les parece útil esta asignatura para su futura profesión de Maestro (ver figura 1), las respuestas fueron: el $65.8 \%$ de los estudiantes la considera una asignatura muy útil (valor 5), un 31.2\% la considera bastante útil (valor 4), 
un $2.7 \%$ la considera medianamente útil (valor 3), y el $0.3 \%$ la considera poco útil (valor 2).

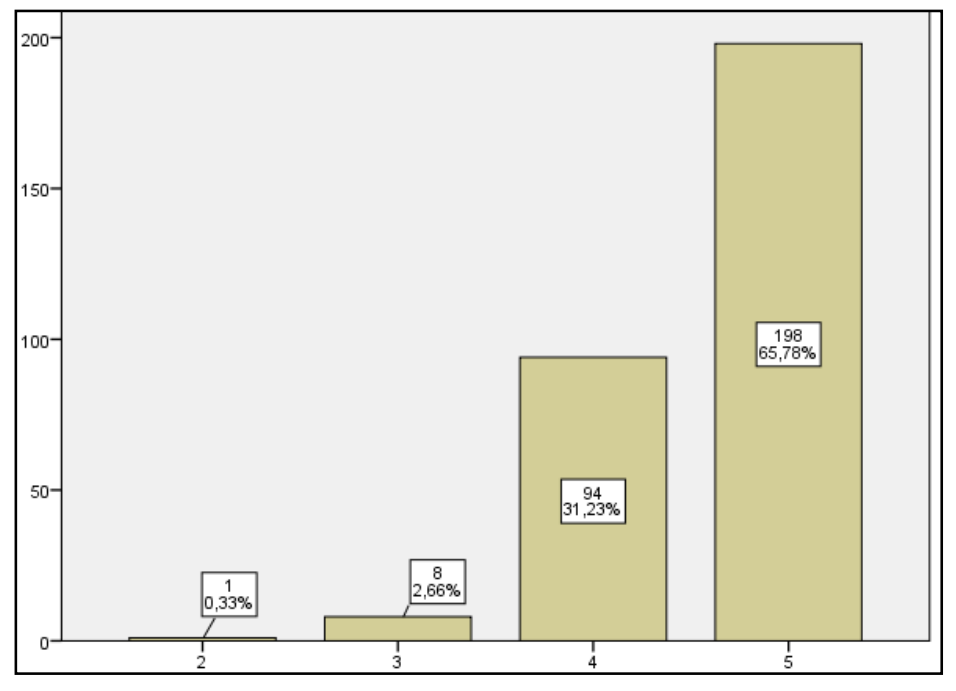

Figura 1. Variable utilidad de la asignatura

\section{Discusión}

De los 301 cuestionarios que los alumnos de primer curso matriculados en "TIC aplicadas a la educación" llenaron, podemos concluir que tal y como señala el informe Universidad 2020 (Fundación Telefónica, 2012), el número de estudiantes mayores de 30 años pudiera haberse duplicado en 10 años debido, entre otras cosas, al envejecimiento demográfico; de esta forma, la mayoría de nuestros alumnos se encuentran en la franja de los 30 a los 35 y de los 24 a los 29 años.

En cuanto al sexo de los encuestados, la gran mayoría (75.4\%) son mujeres, acorde con lo publicado por otras investigaciones que demuestran el predominio de mujeres matriculadas en las titulaciones correspondientes a las ramas sociales y jurídicas (CRUE, 2010).

Como demuestra nuestro estudio, la mayoría de los alumnos matriculados (81.1\%) trabaja actualmente, lo que podría estar relacionado con el mundo digitalizado en el que nos movemos, que está acabando con las barreras geográficas que antes impedían a muchas personas continuar con su formación.

En lo referente a los recursos tecnológicos con los que cuenta el alumno para cursar la asignatura, la mayoría (99.7\%) tiene ordenador con conexión a Internet y el sistema operativo que utilizan los alumnos mayoritariamente es Windows (91.7\%). Este resultado coincide con el informe Universitic 2012 (Crue, 2012) que refleja cómo sólo 1 de cada 4 ordenadores en las universidades españolas tiene un sistema operativo no propietario. Por otro lado, los resultados demuestran una vez más que el acceso a la tecnología ya no es exclusivo de minorías privilegiadas (Fundación Telefónica, 2012b).

Dando a los alumnos la opción de elegir entre el teléfono, el correo electrónico, Skype, el chat o la propia herramienta de videoconferencia del campus, el 93\% ha elegido el teléfono como medio de interacción. Estos datos quizá estén relacionados con que a pesar de que la tecnología está cambiando el modo en que nos comunicamos, el perfil de nuestros alumnos, por edad, aún no se identifica totalmente con el "nativo digital" y faltan aún algunos años para llegar a esto.

Dado que se trata de una universidad online nos interesa conocer la frecuencia de uso de Internet por parte de los alumnos para detectar posibles carencias, a este respecto podemos decir que $93.4 \%$ de los alumnos lo usa a diario. Estos resultados coinciden con lo que nos dice el informe Universidad 2020 (Fundación Telefónica, 2012), según el cual el 97\% de los estudiantes universitarios utiliza Internet de 
forma habitual.

Para poder diseñar un plan de estudios que ayude a que nuestros alumnos puedan hacer frente a las pedagogías emergentes, es necesario amoldarnos a su nivel de conocimientos en materia tecnológica y, en este sentido, el 43.5\% considera que tiene un nivel Intermedio, el 42.2\% dice tener un nivel Básico, un $8 \%$ dice tener nivel Avanzado y sólo el 6.3\% afirma tener un nivel Principiante. Se trata, por tanto, de un grupo heterogéneo de estudiantes en cuanto a competencias tecnológicas, a mitad de camino entre el "nativo digital" (Prensky, 2001) que utiliza la tecnología de manera natural en cualquier campo de su vida cotidiana, y una generación de alumnos predecesores procedentes de la sociedad libresca. Se trata de alumnos que probablemente quieran recibir la información de manera ágil y rápida, y prefieran instruirse de forma lúdica y tecnológica a la manera tradicional, no obstante, aún están adaptándose a este nuevo entorno digital, pues a diferencia de los nativos, no han nacido rodeados de tecnología y mantienen aún ciertas "costumbres" tradicionales (imprimir los materiales del curso, contacto telefónico con los profesores, etc.).

Ahora se espera de los ciudadanos, y de los maestros en particular, una serie de competencias digitales que les permitan desenvolverse con éxito en la sociedad de la información y el conocimiento. En este sentido, preguntamos por el manejo que tienen de los programas básicos que -según la unEsco en sus Estándares de Competencias TIC para docentes (UNESCO, 2008), la Red Universitaria de Tecnología Educativa (RUTE, 2008) o el Libro Blanco del Título Grado en Magisterio de la Agencia Nacional de Evaluación de la Calidad y Acreditación (ANECA, 2005)- los maestros tienen que dominar: los procesadores de textos, la presentación de diapositivas y las hojas de cálculo.

En este sentido, podemos decir que el cuestionario que se aplicó a principios de curso para detectar el perfil de los alumnos, refleja datos que coinciden con el estudio de Espuny, González y Gisbert (2010) y con los desarrollados por Cabero y Llorente (2006) y Selwyn (2008), en cuanto a que son los programas de ofimática los que más conocen y usan los alumnos al entrar en la universidad. De la misma manera, en el estudio de Espuny et al. (2010), queda reflejado cómo la aplicación más popular entre la ofimática es el procesador de textos, seguido del presentador de diapositivas, resultado que coincide con nuestro estudio.

Asimismo, a la pregunta de si han participado en el estudio por cuestiones relacionadas con programas y servicios más específicos, como son los compresores de archivos y los discos duros virtuales, los alumnos respondieron que la mayoría (70.8\%) maneja los compresores; sin embargo también la mayoría de (70.4\%) no emplea discos duros virtuales. Los datos vertidos en nuestro estudio coinciden con la investigación de Espuny et al. (2010) en tanto que los alumnos dominan en menor medida los programas que ganan en especialización.

En cuanto a las aplicaciones más novedosas (blogs, wikis, marcadores sociales,etc.) se concluye que nuestros alumnos dominan en mejor medida los programas tradicionales (procesador de textos, presentador de diapositivas, hoja de cálculo) y desconocen el manejo de las herramienta relacionadas con la Web 2.0, lo que coincide con estudios como el presentado por Espuny et al. (2010). Si bien los medios sociales están penetrando en nuestros alumnos, por el momento no los dominan como para producir, difundir y consumir información y cultura a través de la red, rasgo de las generaciones interactivas (Fundación Telefónica, 2012b).

Además de detectar las competencias tecnológicas de nuestros alumnos mediante este cuestionario diagnóstico, nos hemos propuesto conocer las motivaciones que les han llevado a elegir la carrera docente. En este sentido podemos decir que el principal motivo a la hora de elegir el Grado en Maestro de Primaria ha sido la vocación, pues tienen una alta expectativa hacia las asignaturas en las que aprenden a manejar las TIc y consideran las tecnologías muy útiles para el proceso de enseñar y aprender.

Para finalizar, de los resultados se desgrana la necesidad de un plan de estudios en el Grado en Magisterio que se ajuste a las tendencias pedagógicas propias de la sociedad de la información y el conocimiento, de tal forma que permita a los alumnos adquirir las habilidades para llevar a cabo las tareas de un maestro en un ambiente digital, lo que conlleva el desarrollo de competencias instrumentales, para el uso didáctico 
de la tecnología, para la docencia virtual, así como competencias socioculturales y comunicacionales a través de las Tic (Área, Gutiérrez y Vidal, 2011).

\section{Referencias}

Adell, J. y Castañeda, L. (2012). Tecnologías emergentes, ¿pedagogías emergentes? En J. Hernández, M. Pennesi, D. Sobrino y A. Vázquez (Coords.), Tendencias emergentes en educación con tIC (pp. 13-32). Barcelona: Asociación Espiral, Educación y Tecnología.

Agencia Nacional de Evaluación de la Calidad y de Acreditación. (2005). Libro Blanco. Título Grado de Magisterio (vols. I y II). Madrid: Omán.

Área, M., Gutiérrez, A. y Vidal, F. (2011). Alfabetización digital y competencias informacionales. Madrid: Ariel

Ballester, L. (2001). Bases metodológicas de la investigación educativa. Palma: Universidad de las Islas Baleares.

Bergmann, J. y Sams, A. (2012). Flip your classroom: reach every student in every class every day. Washington, DC: International Society for Technology in Education.

Cabero, J. y Llorente, M. C. (2006). La rosa de los vientos. España: Grupo de Investigación Didáctica.

Cacheiro, M. L. (2011). Recursos educativos TIC de información, colaboración y aprendizaje. Revista PixelBit, 39, 69-81.

Crue. (2012). Universitic 2012. Recuperado de

http://www.crue.org/Publicaciones/Documents/Universitic/2012.pdf

CRUE. (2012b). Tendencias TIC para el apoyo a la docencia universitaria. Recuperado de

http://rua.ua.es/dspace/bitstream/10045/24789/1/Tendencias\%20TIC\%20Docencia\%20\%28web\%20crue \%29.pdf

CRUE. (2010). La universidad española en cifras. Recuperado de http://www.crue.org/Publicaciones/Documents/UEC/LA UNIVERSIDAD ESPANOLA EN CIFRAS.pdf

Espuny, C., González, J. y Gisbert, M. (2010) ¿Cuál es la competencia digital del alumnado al llegar a la universidad? Enseñanza \& Teaching, 28(2), 113-137.

Fox, D. (1989). El proceso de investigación en educación. España: Eunsa.

Fundación Telefónica (2012). Universidad 2020: papel de las TIC en el nuevo entorno socioeconómico. Madrid: Ariel.

Fundación Telefónica (2012b). Aprender con tecnología. Investigación internacional sobre modelos educativos de futuro. Madrid: Ariel.

George, D. y Mallery P. (1995). sPSS/PC+Step by: a simple guide and reference. Belmont, CA.: Wadsworth Publishing Company.

Hernández, O. (2004). Estadística elemental para Ciencias Sociales. Universidad de Costa Rica.

Lage, M. J., Platt, G. J. y Treglia, M. (2000). Inverting the classroom: a gateway to creating an inclusive learning environment. Journal of Economic Education, 31, 30-43. 
Lago, B., Colvin, L. y Cacheiro M. L. (2008). Estilos de aprendizaje y actividades polifásicas: modelo EAAP. Revista Estilos de Aprendizaje, 2, 2-22.

Mishra, P. y Koehler, M. J. (2006). Technological pedagogical content knowledge: a new framework for teacher knowledge. Teachers College Record, 6, 1017-1054.

Nafría, I. (2008). Web 2.0 El usuario, el nuevo rey de Internet. Barcelona: Gestión 2000.

Pardinas, F. (2005). Metodología y técnicas de Investigación en Ciencias Sociales. México: Siglo XXI.

Pino, M. R. y Soto, J. (2010). Identificación del dominio de competencias digitales en el alumnado del grado de magisterio. Revista Teoría de la Educación, 11(3), 336-362.

Prendes, M. P. y Castañeda, L. (2010). Competencias para el uso de tIC en futuros maestros. Comunicar, 35, 175-182.

Prensky, M. (2001). Nativos digitales, inmigrantes digitales. Recuperado de http://files.educunab.webnode.cl/200000062-5aba35bb22/Nativos-digitales-parte1.pdf

Reig, D. y Vílchez, L. F. (2013). Los jóvenes en la era de la hiperconectividad: tendencias, claves y miradas. Madrid: Fundación Telefónica.

Roig, R. y Pascual, L. (2012). Las competencias digitales de los futuros docentes. Un análisis con estudiantes de magisterio de educación infantil de la Universidad de Alicante. @tic, Revista de Innovación Educativa, 9, 53-60.

RUTE. (2008). Declaración de la Junta Directiva de RUTE ante los nuevos títulos universitarios para la formación del profesorado en Educación Infantil, Primaria y Secundaria. Recuperado de http://www.juntadeandalucia.es/averroes/mochiladigital/didactica/Declaracion_RUTE2008.pdf

Selwyn, N. (2008). An investigation of differences in undergraduates' academic use of Internet. Active Learning in Higher Education, 9(1), 11-22.

UNESCO. (2008). Estándares de competencias TIC para docentes. Recuperado de http://www.eduteka.org/pdfdir/UNESCOEstandaresDocentes.pdf 